\title{
El modelo de plan de estudios de la UNCTAD propuesto para el profesional en la Contaduría versus la estructura curricular de las Universidades mexicanas: un estudio comparativo
}

\author{
The curriculum model for the UNCTAD proposed for the Accountancy \\ profession versus the curriculum structure Mexican universities: a \\ comparative study
}

\author{
Gracia Patricia Michel ((pmichelmx@yahoo.com.mx) \\ Universidad de Guadalajara (México)
}

Carmen Fernández-Polvillo (maricarmenfp@euosuna.org)

Escuela Universitaria de Osuna (Universidad de Sevilla)

http://dx.doi.org/10.12795/EDUCADE.2016.i07.02

\begin{abstract}
RESUMEN:
El objetivo de este trabajo es contrastar el modelo propuesto por la UNCTAD contra los Planes de Estudios de las Universidades mexicanas, para identificar aquéllas áreas técnicas de formación en que han cumplido, así como en qué se concentran o qué han descuidado. Los nuevos modelos de negocios, entre otras tendencias, afectan directamente las competencias que el Contador debe ejercer al insertarse en el mercado laboral de forma exitosa. Estas nuevas competencias deben considerarse en un Plan de Estudios. Preocupados por ello la United Nations Conference on Trade and Development (UNCTAD), órgano de la Asamblea de la Organización de las Naciones Unidas, publicó un modelo de Plan de Estudios como una guía y un punto de referencia para señalar los conocimientos técnicos que debe tener un Contador profesional y que debe ser utilizado por las Instituciones de Educación Superior. La muestra se compone de un total de 62 universidades segmentadas según tamaño y tipo de institución. Los resultados, que muestran claramente que en la mayoría de Universidades falta cubrir principalmente en asignaturas optativas, pueden ofrecer un punto de partida a las Universidades mexicanas, que facilite la armonización de sus Planes de Estudio a nivel global.
\end{abstract}

PALABRAS CLAVE: Currículum, Plan de estudios de contador, UNCTAD, Educación del contador, Competencias.

\begin{abstract}
:
The objective of this is paper to contrast the model proposed by UNCTAD against Curricula of the Mexican universities, to identify those technique areas of training that have achieved their goals as well as in what they are concentrated on or what they have neglected. The new businesses models, among other trends directly affect the competences that entry level accountants should bring to the marketplace. Therefore, these new competences should be considered in the accounting curriculum. Concerned about this, the United Nations Conference on Trade and Development (UNCTAD) published a proposed curriculum to serve as guidance to Higher Education Institutions on the technical knowledge that professional accountants should acquire throughout their education. The sample consists of a total of 62 universities segmented by size and type of institution. The results, which clearly show that most universities do not cover the elective subjects, can provide a starting point for Mexican universities, to facilitate the global harmonization of their curricula.
\end{abstract}

Artículo. Recibido: 19-05-16 - Versión revisada: 21-07-16, Aceptado: 01-09-16

Licencia Creative Commons BY NC ND · $2016 \cdot$ Asociación Española de Contabilidad y Administración de Empresas - AECA 
KEYWORDS: Curriculum, Curriculum accountant, UNCTAD, Accountant education, Competencies.

\section{INTRODUCCIÓN}

En los últimos treinta años se han venido presentando cambios constantes en todos los ámbitos y éstos han cambiado profundamente la profesión del Contador Público, quien obligado por un lado al manejo de la información financiera de forma digitalizada con su consecuente adelanto en rapidez y precisión y, por otro, por los requerimientos de información financiera para empresas que han visto globalizar su entorno o se han internacionalizado ellas mismas, obligando al Contador a llevar a cabo su gestión en ambientes internacionales y multiculturales (Boritz, 1999). El profesional de la Contaduría debe responder a los cambios que se presentan en este nuevo entorno laboral, donde se han ido creando nuevas y complejas formas de gestión financiera, ocasionado por la globalización, los mercados de capitales, la competencia, nuevos modelos de negocio, la especialización y tecnologías de información y comunicación que cambian o se crean constantemente.

De todo esto surgen nuevas competencias y, como consecuencia, un nuevo perfil de Contador profesional, con nuevos conocimientos que le permitan una participación activa en la toma de decisiones en todos los campos económicos, lo que supone ahora una preocupación en el espacio educativo: el de un nuevo perfil de egreso de este profesional, que responda a los requerimientos de la práctica profesional (OCDE, 2009), que consiga que sea exitoso, desde el punto de vista de que el egresado logre integrarse en el campo laboral de manera adecuada, cumpliendo con las expectativas del empleador respecto de la profesión (Gómez, Puig, Quirós y Viaño, s.f.). Las Instituciones de Educación Superior (IES) se enfrentan así a nuevas tendencias que afectan el currículum académico, lo que les obliga a renovar sus Planes de Estudio (PE), sino sus egresados verán que su entorno supera sus conocimientos.

Siendo Contaduría la profesión mejor organizada a nivel mundial, sus líderes muestran su preocupación adelantándose a las IES. Un ejemplo de ello es la Federación Internacional de Contadores (International Federation of Accountants/IFAC), que a través de su órgano el Consejo de Normas Internacionales de Formación en Contaduría (International Accounting Education Standards Board/IAESB) ha desarrollado guías para mejorar las normas de formación en contaduría en todo el mundo. El 23 de agosto de 2010, ha publicado el Handbook of International Education Pronouncements, que contiene ocho Normas Internacionales de Formación en Contaduría (IES), originalmente aprobadas en octubre de 2003 y posteriormente revisadas, al igual que tres Declaraciones de Prácticas Internacionales de Formación y entre sus principales objetivos están la armonización de la educación del Contador a nivel mundial, facilitar su movilidad internacional y proveer puntos de referencia que sirvan de medición en la educación del profesional de la Contaduría. Específicamente la IES 2 es la norma que se dedica al Contenido de los programas de formación profesional en contaduría y prescribe su contenido para que los Contadores egresados de las IES sean considerados profesionales.

En el mismo sentido, otro organismo de la IFAC es el Professional Accountants in Business (PAIB), que ha publicado en agosto de 2010 un documento de consulta llamado "Competente y Versátil: cómo los contadores profesionales en empresas impulsan el éxito de la organización sustentable", que motiva a los miembros a hacer aportaciones que le permitan conocer un nuevo perfil deseable del profesional en la Contaduría Pública; igual publicó "Competent and Versatile: Q\&A for Smaller Organizations and the Accountants That Serve Them" en octubre de 2010, que es una 
consulta pública acerca del mismo tema, con el objetivo de conocer qué espera del Contador profesional la sociedad en general.

Otro cuerpo internacional, la United Nations Conference on Trade and Development (UNCTAD), órgano de la Asamblea de la Organización de las Naciones Unidas (ONU), ha incluido la educación del Contador en su agenda desde 1985, mostrando así su preocupación porque éste, como agente de desarrollo económico, tenga los conocimientos técnicos adecuados para cumplir su labor; por ello, en febrero de 1999 publicó un modelo de Plan de Estudios para el Contador profesional, con la intención de ser una guía para el contenido técnico, de tal manera que los Contadores tengan las herramientas técnicas necesarias para la aplicación de sus conocimientos en una economía globalizada; este modelo es un punto de referencia para señalar las competencias técnicas que debe tener un Contador profesional y que debe ser utilizado por las IES si quieren armonizar el perfil de egreso de sus alumnos a nivel global.

En Estados Unidos de Norteamérica, la American Institute of Certified Public Accountants (AICPA) creó en 1999 el Core Competency Framework for Entry into the Accounting Profession, que contempla un conjunto de competencias claves que deben ser presentadas por los Contadores que están ingresando en el mercado de trabajo y que fue elaborado con la opinión de Contadores profesionales lOtt y Barbosa, 2010).

Mientras que en México, el Instituto Mexicano de Contadores Públicos (IMCP) dedicó una edición completa de su revista Contaduría Pública (septiembre 2006) con el fin de mostrar el estado en ese momento de la profesión, de la que podemos rescatar que el Director General de Profesiones de la Secretaría de Educación Pública en México (SEP), en entrevista, señaló que esta institución mostró su preocupación por los PE de la carrera al integrar e instalar la Comisión Técnica Consultiva de Contaduría Pública, "como una instancia de asesoría y apoyo para estudiar y dictaminar temas relativos al ejercicio profesional de la Contaduría Pública"(Meljem, 2006); en la misma edición Romero Fraga (2006) indica que los cambios a los PE de las IES debería ser resultado de un análisis de las exigencias del mercado laboral; por su parte Brambila y Vázquez (2006) en la p. 37 estiman que en el año 2020 los PE deben considerar que "no existen distancias y la colaboración profesional y educativa es mundial" y que las IES deben "fortalecer sus programas de vinculación con el sector productivo y ofrecer planes de estudios acorde a la realidad del mundo del trabajo".

Así mismo, la Comisión de Educación del IMCP, en respuesta a la propuesta del IFAC de las Normas Internacionales de Educación, presentó en diciembre de 2009 el borrador para recibir comentarios de las 8 Normas de Formación para Profesionales en Contaduría (NFPC), proponiendo de esta manera no adoptar la propuesta sino emitir la propia alineada a la de IFAC, objetando que éstas son adecuadas al ámbito educativo y regulatorio mexicano, entre ellas la número 2 se refiere al Contenido de los programas de Educación Profesional en Contaduría que entró en vigor el 1 de enero de 2011, cuyo contenido se reduce a señalar un conjunto de temas muy generalizados, que no nos permitirían lograr el objetivo de este trabajo, en oposición con el de la UNCTAD que hace una serie de señalamientos de contenidos temáticos muy específicos.

También en México la Asociación Nacional de Facultades y Escuelas de Contaduría y Administración (ANFECA), fundada en 1959 y hoy con 274 IES afiliadas, tiene entre sus objetivos básicos el de elaborar conjuntamente planes y programas de estudio, sujetos a las circunstancias regionales, ha ostentado su preocupación por los PE ya que han sido el tema constante en sus Asambleas. 
En el aspecto del fundamento jurídico en México, la Ley General de Educación dedica la sección 2 del Capítulo IV Del Proceso Educativo a los planes y programas de estudio (PE). Específicamente en el artículo 47 regula cómo y qué deben contener éstos, entre otros, en la fracción II que textualmente indica: "Los contenidos fundamentales de estudio, organizados en asignaturas $U$ otras unidades de aprendizaje que, como mínimo, el educando deba acreditar para cumplir los propósitos de cada nivel educativo". Así mismo señala que la Secretaría de Educación, como máximo órgano en materia de educación en México, en el artículo 48 "...realizará revisiones y evaluaciones sistemáticas y continuas de los planes y programas a que se refiere el presente artículo, para mantenerlos permanentemente actualizados". Por lo que podemos observar que en materia legal sólo se regula la forma de elaborar el PE, más no el contenido que se deja libremente a las IES.

Por su parte, los Comités Interinstitucionales para la Evaluación de la Educación Superior (CIEES), en su documento "Guía metodológica para la evaluación de los currícula de licenciatura" (1992), señala que ésta debe estar formada por una serie de aspectos educativos, entre los que se cuenta el PE y que éste, a su vez, debe contener las unidades curriculares, además de la parcelación del conocimiento ordenadas en bloques de formación profesional; la programación de las unidades que incluyan los temarios, los objetos de estudio y la bibliografía; y la secuencia programática, confirmando así lo señalado en el párrafo anterior.

Respecto a las IES mexicanas, la Universidad Nacional Autónoma de México (UNAM), considerada la número uno en este país y la segunda en América latina según el ranking mundial, presentó el pasado mes de agosto de 2011 su nuevo PE, con modificaciones hechas para actualizar su contenido, pero también para considerar la promoción de valores éticos, estéticos y culturales. Entre las modificaciones más importantes está la reducción de nueve a ocho semestres, la participación de actividades de formación integral y la obligación de demostrar inglés intermedio como requisito para la obtención del título.

La Universidad Veracruzana, tercera en México en número de alumnos en Contaduría, también modificó SU PE para el periodo escolar 2011/2012, con un estudio previo realizado por su Comisión de Evaluación de Plan de Estudios, disminuyendo el número de créditos de 380 a 324, los créditos electivos de 19 a 16, agregando con peso en 6 créditos la acreditación del idioma inglés. El PE anterior se venía aplicando desde 2003.

En este contexto, podemos observar que, a pesar de que existen estudios y acciones al respecto, encontramos limitaciones para que directamente las IES determinen con seguridad un perfil de egreso de estos profesionales, actual y puntual, ya que éstas deben cumplir no sólo con lo que IFAC pide, sino con lo que el mercado laboral en su entorno necesita, también su propia normatividad interna, los organismos reguladores gubernamentales respecto del cumplimiento de obligaciones legales, las asociaciones de IES, las agrupaciones profesionales internacionales, nacionales, regionales o locales y la sociedad en general que espera profesionistas que apoyen el desarrollo sustentable de las naciones.

Por todo lo anterior expuesto, hemos detectado que en respuesta realmente objetiva a la necesidad mundial de conseguir ese nuevo perfil de egreso, la UNCTAD es quién nos hace la propuesta más concreta en la publicación de un modelo del PE, elaborado por un grupo de especialistas en normas internacionales de contabilidad denominado Intergovernmental Group of Experts on International Standards of Accounting and Reporting (ISAR), formado por órganos gubernamentales y no gubernamentales, siendo el único órgano intergubernamental la ONU. Por esta 
importante razón consideraremos este modelo como objeto de estudio para este trabajo, contrastándolo contra los PE de las IES mexicanas.

Como antecedentes de dicho modelo tenemos que durante el $15^{\circ}$ periodo de Sesiones de este organismo, se acordó que se dedicara el $16^{\circ}$ periodo de Sesiones a "la elaboración de un programa mundial de estudios de contabilidad y otras normas y requisitos de cualificación" (UNCTAD, 1999) y dado que se consideró que los estudios al respecto estaban muy avanzados "era necesario preparar un programa mundial que sirviera de referencia y permitiese reducir el tiempo y el costo de negociar acuerdos de reconocimiento mutuo para los contables profesionales", se le pidió a la UNCTAD que, en cooperación con otros organismos, llevara a cabo la elaboración de un Plan de Estudios que se pudiera aplicar a nivel mundial.

En respuesta a ésto, resultó la creación de un documento denominado Guideline on national requeriments for the qualification of the professional accountants; este documento hace un señalamiento muy minucioso de los requerimientos técnicos, actitudes y valores necesarios para la educación del Contador profesional, así mismo indica que se necesita práctica profesional, educación continua y certificación de capacidades.

Posteriormente revisado en dos talleres, en abril y junio de 2003, fue presentado en la Vigésima Sesión y fue aprobado el modelo de Plan de Estudios y, actualmente, está siendo ultimado junto con la IFAC (UNCTAD, 2003); señalándose en el Informe del Grupo de Trabajo Intergubernamental de Expertos en Normas Internacionales de Contabilidad y Presentación de Informes sobre su Vigésimo Periodo de Sesiones que este modelo "es sólo una parte de uno más amplio que ha de servir de referencia para la cualificación de los contadores profesionales".

Este trabajo se enfoca únicamente en el contraste del contenido técnico de formación de este modelo, contra el contenido de los Planes de Estudio de una muestra de IES mexicanas, por lo cual es necesario señalar que, analizando el modelo propuesto por la UNCTAD, encontramos que su estructura curricular tiene cuatro clasificaciones básicas, a su vez cada una de estas clasificaciones contiene módulos y éstos contienen los temas que lo forman y luego, en incisos, se hace una relación detallada de los temas que deben ser el contenido temático de cada módulo, dichos temas nos permitieron clasificar las asignaturas de los PE de las IES mexicanas.

Por razones prácticas nuestro trabajo hará un contraste sólo hasta el segundo nivel de cada módulo, por lo quedará como sigue:

1. Conocimiento de la Organización y la Actividad Comercial, que en el trabajo llamamos por abreviar Organización y Negocios.

\subsection{Economía.}

1.2 Estadísticas empresariales.

1.3 La Empresa (Dirección).

1.4 Administración (Operaciones y Recursos Humanos).

1.5 Mercadotecnia.

1.6 Negocios Internacionales.

2. Tecnologías de la Información.

2.1 Tecnologías de la Información. 
El modelo de plan de estudios de la UNCTAD propuesto para el profesional en la Contaduría versus la estructura curricular de las Universidades mexicanas: un estudio comparativo

3. Conocimientos Básicos de Contabilidad, Auditoria, Imposición Fiscal y sectores relacionados con la Contabilidad (que llamamos Conocimientos básicos Contabilidad, Auditoria y Tributación).

3.1 Contabilidad Básica.

3.2 Contabilidad Financiera.

3.3 Contabilidad Avanzada.

3.4 Contabilidad de Gestión (Costos/Costes).

3.5 Tributación.

3.6 Sistemas de Información Contable.

3.7 Derecho.

3.8 Auditoria Básica.

3.9 Finanzas.

3.10 Integración del Conocimiento.

4. Nivel optativo (avanzado) de Contabilidad, Finanzas y conocimientos afines (Electivas avanzadas Contabilidad, Finanzas y relacionadas).

4.1 Contabilidades Especializadas.

4.2 Contabilidad de Gestión (Costos/Costes).

4.3 Tributación.

4.4 Derecho.

4.5 Auditoria.

4.6 Finanzas.

4.7 Prácticas.

Es preciso indicar que los nombres de los temas de cada módulo no son traducción exacta del inglés en el que está escrito el documento original, por razones puramente prácticas, sin embargo se conserva la esencia de los sub-temas que la forman.

Por todo lo anterior expuesto, nos preguntamos si en México los PE contienen los temas señalados en el Modelo propuesto por la UNCTAD, por lo que nos cuestionamos también en qué se centran, qué han descuidado y cuál dispersión tienen. De igual manera nos cuestionamos acerca del nivel de cumplimiento con este modelo por tipo de IES, si cumplen en mayor o menor grado las IES públicas o las privadas o si lo hacen por tamaño de IES, considerando para ello el número de alumnos matriculados.

Este trabajo forma parte de una tesis doctoral más amplia, cuya línea de investigación es un estudio empírico del perfil deseable de egreso del profesional en la Contaduría en México, en el que pretendemos determinar las características deseables del nuevo perfil del Contador profesional. En este trabajo hacemos un avance en los conocimientos técnicos.

Versiones anteriores de este trabajo se han presentado en el XV Congreso Internacional sobre Innovaciones en Docencia e Investigación en Ciencia Económico Administrativas en septiembre de 2012, evento organizado por Asociación de Profesores de Contaduría y Administración de México, A.C., y en el XVII Congreso 
Internacional de Contaduría, Administración e Informática patrocinado por la Universidad Nacional Autónoma de México en octubre de 2012. Los comentarios recibidos en dichos eventos nos han permitido hacer cambios con el fin de lograr el objetivo de este trabajo.

Trabajos similares a éste, muestran que Oliveira, Veneroso y Carvalho de Souza (2009) en un contraste del modelo de la UNCTAD contra 11 Universidades brasileñas del Estado de Santa Catarina, encontraron un $88.27 \%$ de analogía con el modelo, a pesar de que sólo cuatro de las Universidades de la muestra ofertan Contabilidad Internacional como asignatura obligatoria. Otro ejemplo es el de Ott y Barbosa (2010), también de Brasil, que reconocieron la necesidad de fortalecer los PE con asignaturas propias a Organización y Negocios, ya que la demanda del mercado de trabajo es para profesionales capaces de participar activamente en la toma de decisiones.

\section{METODOLOGÍA}

Para poder responder a las preguntas de investigación y en base a información documental encontrada, realizamos la investigación en las siguientes etapas:

1. Con el fin de conocer a cuánta y a qué información sobre PE nos enfrentaríamos en México, procedimos primero a crear una base de datos que incluyera a absolutamente todos los establecimientos de las IES que ofertan la carrera de Contaduría, partiendo de datos publicados por la Secretaría de Educación Pública (SEP), en su página web oficial, sobre la matrícula en IES para el periodo escolar $2011 / 2012$, clasificándola con todos los datos identificables proporcionados por la SEP. En esta etapa nos encontramos con que en 1,067 establecimientos de un total de 625 IES, se oferta la carrera de Contaduría, haciendo un total de 142,530 alumnos matriculados para dicho periodo escolar.

2. Como resultado del análisis de la base de datos anterior, se procedió a tomar una muestra de IES, consistente en 63 de ellas que representan tanto a las IES públicas en sus distintos tipos, Institutos Tecnológicos y a las IES privadas, dividiendo estas últimas en IES con más de mil alumnos y con menos de mil alumnos, dada la gran diferencia en matrícula que encontramos en ellas, observando que la gran mayoría tienen menos de mil alumnos, por lo que el resultado es una división de cuatro bloques de información formado por 20 IES públicas, 15 Institutos Tecnológicos, 8 IES privadas con más de mil alumnos y 20 IES privadas con menos de mil alumnos (tabla 1). La clasificación de las IES por la SEP indica que las IES públicas pueden ser autónomas $O$ no autónomas y federales $O$ estatales 0 bien Universidades Tecnológicas, pero al depender todas de presupuesto públicos, las hemos clasificado simplemente como públicas, ya que consideramos que esas condiciones no afectan la forma de construir su PE, ya que todas lo hacen de forma libre y autónoma.

3. Como siguiente paso se procedió a obtener el PE de cada IES de la muestra, para el periodo escolar 2011/2012 con oferta escolarizada, consiguiendo en un primer paso el dato directamente de la web de cada IES, después solicitando vía e-mail la información faltante y como último recurso haciéndolo vía telefónica; en esta etapa de la investigación se eliminó a aquellas IES que no publican SU PE ni es posible la comunicación con ellas, dado la escasa información que publican en su web, sustituyéndolas por otras IES para mantener el mismo número en la muestra, exceptuando al grupo de IES privadas de más de mil alumnos, ya que disminuyó en una IES al no encontrarse más con esas características, dejando la muestra final en 62 IES. 
El modelo de plan de estudios de la UNCTAD propuesto para el profesional en la Contaduría versus la estructura curricular de las Universidades mexicanas: un estudio comparativo

4. Como resultado del análisis de los PE, en seguida se realizaron catorce cédulas sobre las áreas de estudio comunes en los Planes de Estudios de las IES en México, por tipo de IES: Administración, Auditoria, Contabilidad, Costos, Derecho, Economía, Finanzas, Fiscal, Herramientas, Idiomas, Informática, Matemáticas, Asignaturas Terminales y Valores y Actitudes.

5. El dato que nos servirá de contraste será el número de asignaturas de cada IES en el $\mathrm{PE}$, clasificado por área de estudio y por tipo de IES.

6. Con el fin de poder contrastar con el modelo publicado por la UNCTAD, se analizó éste observando que la UNCTAD, en el modelo desarrollado, hace cuatro clasificaciones por lo que procedimos a realizar cuatro cédulas siguiendo esta clasificación, por cada una de las áreas de estudio, tomando como referencia el número de asignaturas destinado por cada IES y, a su vez, cada una de ellas la subdividimos en las cuatro clasificaciones de la muestra, más una por el total de la muestra. Los temas de cada cédula son los módulos señalados en la Introducción.

Tabla 1. Descripción de la muestra (número de instituciones y alumnos por tipo)

Panel A. Número de instituciones

\begin{tabular}{|c|c|c|c|c|c|c|}
\hline & Total & Inicial & Elimin. & Sustituidas & $\begin{array}{c}\text { Muestra } \\
\text { final }\end{array}$ & $\%$ \\
\hline Universidades Públicas & 33 & 20 & 1 & 1 & 20 & 60.61 \\
\hline Institutos Públicos & 65 & 15 & 3 & 3 & 15 & 23.08 \\
\hline Universidades Privadas +1000 alumnos & 8 & 8 & 1 & 0 & 7 & 87.5 \\
\hline Universidades Privadas - 1000 alumnos & 519 & 20 & 2 & 2 & 20 & 3.85 \\
\hline SUMAS & 625 & 63 & 7 & 6 & 62 & 9.92 \\
\hline
\end{tabular}

Panel B. Total alumnos matriculados

\begin{tabular}{|c|c|c|c|}
\hline & Total & Muestra & $\%$ \\
\hline Universidades Públicas & 79,789 & 68,726 & 86.13 \\
\hline Institutos Públicos & 18,484 & 8,610 & 46.58 \\
\hline Universidades Privadas +1000 alumnos & 11,495 & 10,107 & 87.93 \\
\hline Universidades Privadas - 1000 alumnos & 32,762 & 8,559 & 26.12 \\
\hline SUMAS & 142,530 & 96,002 & 67.36 \\
\hline
\end{tabular}

Nota: la muestra no considera las IES privadas de -1000 alumnos que ofertan la carrera pero no tienen alumnos matriculados.

\section{RESULTADOS}

Analizando los resultados obtenidos (ver tablas 2 y 3) hemos elaborado una serie de observaciones en el contraste del modelo de PE de la UNCTAD con las IES mexicanas, entre las que podemos destacar:

1. Organización y Negocios:

a. Encontramos aquí que todos los tipos de IES dedican un gran número de asignaturas a la Empresa, que se refiere a Dirección de Empresas, mientras que las Universidades e Institutos Públicos dedican el mayor número de asignaturas a Estadísticas empresariales. 
b. En el caso de Negocios Internacionales, la asignatura más descuidada por todas, tan sólo el $7 \%$ de los Institutos públicos la imparte y la imparten en general sólo el $21 \%$ de todas las IES de la muestra.

c. Encontramos que en las asignaturas del módulo La Empresa es donde hay mayor variación entre mínimos y máximos, esto significa que mientras que algunas IES dedican un gran número de asignaturas al módulo, otras un mínimo o ninguna. Otro ejemplo de variación se da en las Universidades públicas, donde la media del módulo Economía es 2.85 pero en una IES le dedican 8 asignaturas.

La asignatura Estadísticas Empresariales, denominada por la UNCTAD "Módulo sobre los métodos cuantitativos y las estadísticas de la actividad comercial", debería comprender todas las materias de matemáticas, entre las que se encuentra el cálculo del interés simple y compuesto por ejemplo, que en muchas IES se oferta en el contenido temático de alguna asignatura de Finanzas y ésto afecta el resultado de este módulo ya que Finanzas la encontramos en el número 3.

2. Tecnologías de la Información:

a. En este módulo encontramos que la media más alta se encuentra en el grupo de los Institutos públicos que es de 2.33 y la más baja en las Universidades privadas de más de mil alumnos donde es 1, resultado que nos indica que en los Institutos públicos se hace más énfasis en la preparación en esta área, pero que también puede ser que se haya detectado que es necesario así, ya que la oferta de esta asignatura depende de los requisitos de ingreso de cada IES.

b. Mientras que en todas las Universidades públicas se oferta TIC's y en la mayoría de los Institutos, en las IES privadas baja hasta el $70 \%$, indicándonos que una gran cantidad de IES privadas no ofertan ninguna asignatura de Tecnologías de Información.

c. Dentro de este módulo la UNCTAD considera el comercio electrónico y las IES mexicanas la ofertan como parte de la asignatura Mercadotecnia, excepto la Universidad Autónoma de Chihuahua que la oferta como tal.

3. Conocimientos básicos: Contabilidad, Auditoria y Tributación:

a. La media general en Contabilidad Básica es de 1.13 que es razonable para los temas propuestos por la UNCTAD.

b. Una de las asignaturas menos ofertada (cubierta) es Contabilidad Avanzada, sin embargo hemos observado que ésta se ofrece como electiva en la mayoría de las IES, que resulta congruente con la sugerencia de la UNCTAD.

c. Las asignaturas con más diferencia entre mínimos y máximos en las IES privadas es Finanzas, detectándose un mínimo de 1 asignatura y un máximo de 9, cuando la media es de casi 5.5 en los dos tipos de IES privadas. Sin embargo en las Universidades públicas es Derecho y en los Institutos es Tributación las que muestran mayores diferencias.

d. Especial atención pusimos a Tributación, al tratarse de una asignatura que en trabajos similares a éste se señala como asignatura demandada por el público (Arquero, Donoso-Anes, Jiménez-Cardoso y González, 2009), encontrando que es muy ofertada, dado que la media en Universidades públicas es de 4.6, en Institutos públicos es de 3.5, en IES privadas de más de 1000 alumnos 4.7 y en menos de 1000 alumnos es de 5; observándose que en las IES de menos alumnos se oferta más esta asignatura. 
El modelo de plan de estudios de la UNCTAD propuesto para el profesional en la Contaduría versus la estructura curricular de las Universidades mexicanas: un estudio comparativo

e. Con excepción a Institutos públicos, coincide que finanzas es el grupo de asignaturas más ofertadas por el resto.

f. En el caso de Integración del Conocimiento la media general es de 0.9 dándose los niveles más bajos de media entre las IES privadas.

El caso de Integración del Conocimiento, considerado como "un punto culminante" por la UNCTAD y que tiene por objetivo "exponer los procesos de aprendizaje que estimulan y fortalecen la incorporación de la capacidad de razonamiento intelectual, las comunicaciones verbales y escritas y las aptitudes de relación interpersonal...", recomendándose se oferte en el último periodo escolar con el fin de que el alumno integre los conocimientos adquiridos en las diferentes asignaturas, detectamos que en las IES diferentes a Institutos públicos existe alguna actividad para integrar los conocimientos adquiridos, pero al no darle un peso en créditos no fue posible realizar un contraste con ellas, o la(s) actividad(es) se integran a alguna asignatura dentro de su contenido temático; en estos casos hemos detectado que algunas IES señalan como requisito de titulación pero sin asignar créditos a alguna actividad de integración del conocimiento, tales como prácticas profesionales. Es necesario señalar que los Institutos públicos tienen integrado en su PE una residencia profesional con una asignación media de créditos de 15.9, por lo que se puede identificar claramente esta actividad. No se incluyó el Servicio Social como una actividad integradora, por tratarse de una asistencia de reciprocidad a la sociedad y que la mayoría de las veces en ella el alumno no lleva a cabo funciones propias de su carrera.

4. Electivas: Contabilidad, Finanzas y relacionadas:

a. En los siete grupos temáticos que forman este módulo, lo más sobresaliente es la escasez de oferta en todos los grupos; en las Universidades públicas lo que menos se oferta es Contabilidad de Gestión y Derecho; en los Institutos públicos sólo se oferta Auditoria y Finanzas y no llega a 1 la media; en las IES privadas de más de 1000 alumnos no se oferta Finanzas y del resto la media no llega a 0.5 y en las IES privadas de menos de 1000 alumnos no hay oferta en Derecho y del resto apenas llega a 0.5 la media. En ningún grupo de IES se ofertan prácticas de manera electiva.

b. Las asignaturas más ofertadas se dan en Universidades públicas y, con mucha diferencia, en IES privadas de menos de mil alumnos.

c. El $20 \%$ de las Universidades públicas no hacen ofertas de electivas, el $86 \%$ de los Institutos tampoco, ni el $85 \%$ de las IES privadas de más de 1000 alumnos, así como el $80 \%$ de las de menos de 1000 alumnos. En este caso sabe señalar que la Universidad Nacional Autónoma de México es quién hace mayor número de oferta de electivas, seguida de la Universidad de Guadalajara y la Universidad Autónoma de San Luis Potosí, todas en el grupo de Universidades públicas. 
Tabla 2. Observaciones al contrate entre el modelo de Plan de Estudios de la UNCTAD y las Universidades mexicanas.

Panel A. Organización y Negocios

\begin{tabular}{|c|c|c|c|}
\hline UNIVERSIDADES PÚBLICAS & INSTITUTOS PÚBLICOS & $\begin{array}{c}\text { UNIV. PRIVADAS +1000 } \\
\text { ALUMNOS }\end{array}$ & $\begin{array}{c}\text { UNIV. PRIVADAS - } 1000 \\
\text { ALUMNOS }\end{array}$ \\
\hline $\begin{array}{l}\text { - En todas las IES se } \\
\text { imparte Economía. } \\
\text { - La asignatura más } \\
\text { cubierta es Estadísticas } \\
\text { Empresariales. } \\
\text { - De } 6 \text { áreas de } \\
\text { conocimientos hay } \\
\text { una media de } 4.85 \text { de } \\
\text { asignaturas cubiertas. } \\
\text { - El } 70 \% \text { no cubren } \\
\text { Negocios } \\
\text { Internacionales. } \\
\text { - Las asignaturas con } \\
\text { más diferencia de } \\
\text { oferta entre mínimos y } \\
\text { máximos son: la } \\
\text { Empresa, Economía y } \\
\text { Estadísticas } \\
\text { Empresariales. } \\
\text { - En Economía la media } \\
\text { es } 2.85, \text { pero en una IES } \\
\text { se ofertan } 8 \\
\text { asignaturas. } \\
\text { - El número máximo de } \\
\text { asignaturas en un solo } \\
\text { tema es } 13 \text { y es la } \\
\text { Empresa. }\end{array}$ & $\begin{array}{l}\text { - En todas las IES se } \\
\text { imparte Economía, } \\
\text { Estadísticas } \\
\text { Empresariales y La } \\
\text { Empresa. } \\
\text { - La asignatura más } \\
\text { cubierta es Estadísticas } \\
\text { Empresariales. } \\
\text { - De } 6 \text { áreas de } \\
\text { conocimientos hay } \\
\text { una media de } 4.68 \text { de } \\
\text { asignaturas cubiertas. } \\
\text { - El 93\% no cubren } \\
\text { Negocios } \\
\text { Internacionales. } \\
\text { - Las asignaturas con } \\
\text { más diferencia de } \\
\text { oferta entre mínimos y } \\
\text { máximos son: la } \\
\text { Empresa y } \\
\text { Administración. } \\
\text { - En la Empresa la media } \\
\text { es } 2.6, \text { pero en una IES } \\
\text { se ofertan } 6 \\
\text { asignaturas. } \\
\text { - El número máximo de } \\
\text { asignaturas en un solo } \\
\text { tema es } 6 \text { y es la } \\
\text { Empresa. }\end{array}$ & $\begin{array}{l}\text { - En todas las IES se } \\
\text { imparte Economía, La } \\
\text { Empresa y } \\
\text { Administración. } \\
\text { - La asignatura más } \\
\text { cubierta es La } \\
\text { Empresa. } \\
\text { - De } 6 \text { áreas de } \\
\text { conocimientos hay } \\
\text { una media de } 4.71 \text { de } \\
\text { asignaturas cubiertas. } \\
\text { - El } 71 \% \text { no cubren } \\
\text { Negocios } \\
\text { Internacionales } \\
\text { - Las asignaturas con } \\
\text { más dife-rencia de } \\
\text { oferta entre mínimos y } \\
\text { máximos son: } \\
\text { Estadísticas } \\
\text { Empresariales y la } \\
\text { Empresa. } \\
\text { - En la Empresa la media } \\
\text { es } 3.28 \text { pero en una IES } \\
\text { se ofertan } 6 \\
\text { asignaturas. } \\
\text { - El número máximo de } \\
\text { asig-naturas en un solo } \\
\text { tema es } 6 \text { y es } \\
\text { Estadísticas } \\
\text { Empresariales y en la } \\
\text { Empresa. }\end{array}$ & $\begin{array}{l}\text { - En todas las IES se } \\
\text { imparte Economía y } \\
\text { La Empresa. } \\
\text { - La asignatura más } \\
\text { cubierta es La } \\
\text { Empresa. } \\
\text { - De } 6 \text { áreas de } \\
\text { conocimientos hay } \\
\text { una media de } 4.3 \text { de } \\
\text { asignaturas cubiertas. } \\
\text { - El } 80 \% \text { no cubren } \\
\text { Negocios } \\
\text { Internacionales. } \\
\text { - Las asignaturas con } \\
\text { más diferencia de } \\
\text { oferta entre mínimos y } \\
\text { máximos son: } \\
\text { Economía, Estadísticas } \\
\text { Empresariales y la } \\
\text { Empresa. } \\
\text { - En la Empresa la } \\
\text { media es } 5,4, \text { pero en } \\
\text { una IES se ofertan } 13 \\
\text { asignaturas. } \\
\text { - El número máximo de } \\
\text { asignaturas en un solo } \\
\text { tema es } 13 \text { y es la } \\
\text { Empresa. }\end{array}$ \\
\hline
\end{tabular}

Panel B. Tecnologías de la Información

\begin{tabular}{|c|c|c|c|}
\hline UNIVERSIDADES PÚBLICAS & INSTITUTOS PÚBLICOS & $\begin{array}{l}\text { UNIV. PRIVADAS +1000 } \\
\text { ALUMNOS }\end{array}$ & $\begin{array}{l}\text { UNIV. PRIVADAS - } 1000 \\
\text { ALUMNOS }\end{array}$ \\
\hline $\begin{array}{l}\text { - En todas las IES se } \\
\text { imparte. } \\
\text { - La media de } \\
\text { asignaturas es } 1.65\end{array}$ & $\begin{array}{l}\text { - En } 93 \% \text { de IES se } \\
\text { imparte. } \\
\text { - La media de } \\
\text { asignaturas es } 2.33\end{array}$ & $\begin{array}{l}\text { - En } 71.4 \% \text { de IES se } \\
\text { imparte. } \\
\text { - La media de } \\
\text { asignaturas es } 1\end{array}$ & $\begin{array}{l}\text { - En } 70 \% \text { de IES se } \\
\text { imparte. } \\
\text { •La media de } \\
\text { asignaturas es } 1.35\end{array}$ \\
\hline
\end{tabular}


El modelo de plan de estudios de la UNCTAD propuesto para el profesional en la Contaduría versus la estructura curricular de las Universidades mexicanas: un estudio comparativo

Panel C. Conocimientos básicos

\begin{tabular}{|c|c|c|c|}
\hline UNIVERSIDADES PÚBLICAS & INSTITUTOS PÚBLICOS & $\begin{array}{c}\text { UNIV. PRIVADAS +1000 } \\
\text { ALUMNOS }\end{array}$ & $\begin{array}{c}\text { UNIV. PRIVADAS - } 1000 \\
\text { ALUMNOS }\end{array}$ \\
\hline $\begin{array}{l}\text { - La media en } \\
\text { contabilidad básica es } \\
1.3 \\
\text { - Las materias menos } \\
\text { cubiertas son: } \\
\text { Contabilidad básica, } \\
\text { Sistemas de } \\
\text { Información Contable } \\
\text { e Integración del } \\
\text { Conocimiento. } \\
\text { - La menos cubierta es } \\
\text { Contabilidad } \\
\text { avanzada. } \\
\text { - La media de materias } \\
\text { cubiertas es } 9.15 \\
\text { - La asignatura con más } \\
\text { diferencia entre } \\
\text { mínimos y máximos es } \\
\text { Derecho. } \\
\text { - Con una media en } \\
\text { Tributación de } 4.6, \text { el } \\
15 \% \text { de las IES ofertan } 6 \\
\text { asignaturas y el } 20 \% 3 . \\
\text { La moda es } 5 . \\
\text { Contabilidad } \\
\text { Financiera, Derecho y } \\
\text { Finanzas son las } \\
\text { asignaturas más } \\
\text { ofertadas. } \\
\end{array}$ & $\begin{array}{l}\text { - La media en } \\
\text { contabilidad básica es } \\
\text { 1. } \\
\text { - Las materias menos } \\
\text { cubiertas son: } \\
\text { Contabilidad básica, } \\
\text { Contabilidad } \\
\text { Avanzada y Sistemas } \\
\text { de Información } \\
\text { Contable. } \\
\text { - La menos cubierta es } \\
\text { Contabilidad } \\
\text { avanzada. } \\
\text { - La media de materias } \\
\text { cubiertas es } 9.07 \\
\text { - La asignatura con más } \\
\text { diferencia entre } \\
\text { mínimos y máximos es } \\
\text { Tributación. } \\
\text { - Con una media en } \\
\text { Tributación de } 3.5, \text { una } \\
\text { IES oferta } 7 \text { asignaturas } \\
\text { y el } 80 \% 3 \text {. La moda es } \\
\text { 3. } \\
\text { - Contabilidad } \\
\text { Financiera, Tributación } \\
\text { y Auditoria básica son } \\
\text { las asignaturas más } \\
\text { ofertadas. }\end{array}$ & $\begin{array}{l}\text { - La media en } \\
\text { contabilidad básica es } \\
1.1 \\
\text { - Las materias menos } \\
\text { cubiertas son: } \\
\text { Contabilidad básica, } \\
\text { Contabilidad } \\
\text { Avanzada e } \\
\text { Integración del } \\
\text { Conocimiento. } \\
\text { - La menos cubierta es } \\
\text { Integración del } \\
\text { Conocimiento. } \\
\text { - La media de materias } \\
\text { cubiertas es } 8.14 \\
\text { - La asignatura con más } \\
\text { diferencia entre } \\
\text { mínimos y máximos es } \\
\text { Finanzas. } \\
\text { - Con una media en } \\
\text { Tributación de } 4.7 \text {, una } \\
\text { IES oferta } 7 \text { asignaturas } \\
\text { y el } 28.6 \% 3 \text {. La moda } \\
\text { es } 5 . \\
\text { - Finanzas y Tributación } \\
\text { son las asignaturas más } \\
\text { ofertadas. }\end{array}$ & $\begin{array}{l}\text { - La media en } \\
\text { contabilidad básica es } \\
1.05 \\
\text { - Las materias menos } \\
\text { cubiertas son: } \\
\text { Contabilidad básica, } \\
\text { Contabilidad } \\
\text { Avanzada e } \\
\text { Integración del } \\
\text { Conocimiento. } \\
\text { - La menos cubierta es } \\
\text { Integración del } \\
\text { Conocimiento. } \\
\text { - La media de materias } \\
\text { cubiertas es } 8.65 \\
\text { - La signatura con más } \\
\text { diferencia entre } \\
\text { mínimos y máximos es } \\
\text { Finanzas. } \\
\text { - Con una media en } \\
\text { Tributación de } 5 \text {, el } \\
\text { 10\% ofertan } 7 \\
\text { asignaturas y el } 5 \% 2 . \\
\text { La moda es } 6 . \\
\text { - Finanzas y } \\
\text { Contabilidad } \\
\text { Financieras son las } \\
\text { asignaturas más } \\
\text { ofertadas. }\end{array}$ \\
\hline
\end{tabular}

Panel D. Electivas en conocimientos avanzados

\begin{tabular}{|c|c|c|c|}
\hline UNIVERSIDADES PÚBLICAS & INSTITUTOS PÚBLICOS & $\begin{array}{l}\text { UNIV. PRIVADAS +1000 } \\
\text { ALUMNOS }\end{array}$ & $\begin{array}{l}\text { UNIV. PRIVADAS - } 1000 \\
\text { ALUMNOS }\end{array}$ \\
\hline $\begin{array}{l}\text { - Las asignaturas menos } \\
\text { cubiertas son: } \\
\text { Contabilidad de } \\
\text { Gestión, Derecho y } \\
\text { Prácticas. } \\
\text { - La asignatura mejor } \\
\text { cubierta es } \\
\text { Contabilidades } \\
\text { Especiales. } \\
\text { - Ninguna IES oferta } \\
\text { Prácticas. } \\
\text { - La media en } 7 \text { temas } \\
\text { es } 3.25 \text {. } \\
\text { - El } 20 \% \text { de las IES no } \\
\text { ofertan electivas. } \\
\text { - Mientras que el } 15 \% \text { de } \\
\text { las IES ofertan más de } \\
10 \text { asignaturas en } \\
\text { Contabilidades } \\
\text { Especiales, el } 30 \% \text { no } \\
\text { oferta ninguna. La } \\
\text { máxima es } 27 .\end{array}$ & $\begin{array}{l}\text { - Las asignaturas menos } \\
\text { cubiertas son todas, } \\
\text { excepto: Auditoria y } \\
\text { Finanzas. } \\
\text { - La asignatura mejor } \\
\text { cubierta es Auditoria. } \\
\text { - Ninguna IES oferta } \\
\text { Prácticas. } \\
\text { - La media en } 7 \text { temas } \\
\text { es } 0.13 \\
\text { - El } 86.7 \% \text { de las IES no } \\
\text { ofertan electivas. } \\
\text { - No hay oferta en } \\
\text { Contabilidades } \\
\text { Especiales. }\end{array}$ & $\begin{array}{l}\text { - Las asignaturas menos } \\
\text { cubiertas son: Finanzas } \\
\text { y Prácticas. } \\
\text { - La asignatura mejor } \\
\text { cubierta es } \\
\text { Contabilidades } \\
\text { Especiales. } \\
\text { - Ninguna IES oferta } \\
\text { Prácticas. } \\
\text { - La media en } 7 \text { temas } \\
\text { es } 0.714 \\
\text { - El } 85.7 \% \text { de las IES no } \\
\text { ofertan electivas. } \\
\text { - Sólo en una IES de la } \\
\text { muestra se oferta } \\
\text { Contabilidades } \\
\text { Especiales, ofreciendo } \\
2 \text { asignaturas. }\end{array}$ & $\begin{array}{l}\text { - Las asignaturas menos } \\
\text { cubiertas son: Derecho } \\
\text { y Prácticas. } \\
\text { - La asignatura mejor } \\
\text { cubierta es Tributación. } \\
\text { - Ninguna IES oferta } \\
\text { Prácticas. } \\
\text { - La media en } 7 \text { temas } \\
\text { es } 0.6 \\
\text { - El } 80 \% \text { de las IES no } \\
\text { ofertan electivas. } \\
\text { - Sólo en dos IES de la } \\
\text { muestra, que } \\
\text { representa el } 10 \% \text { se } \\
\text { ofertan asignaturas de } \\
\text { Contabilidades } \\
\text { Especiales, siendo la } \\
\text { máxima } 6 \text {. }\end{array}$ \\
\hline
\end{tabular}


Gracia Patricia Michel, Carmen Fernández-Polvillo

El modelo de plan de estudios de la UNCTAD propuesto para el profesional en la Contaduría versus la estructura curricular de las Universidades mexicanas: un estudio comparativo

Tabla 3. Contraste de plan de estudios IES mexicanas contra UNCTAD matrícula 2011/2012

Número de asignaturas media por tipo de IES

\begin{tabular}{|c|c|c|c|c|c|c|c|c|c|c|c|c|c|}
\hline & OINヨIWITdWחO $\forall I O \exists W$ & & & $\stackrel{2}{\wedge}$ & & & $\overline{0}$ & & & 8 & & & 命 \\
\hline \multirow{7}{*}{ 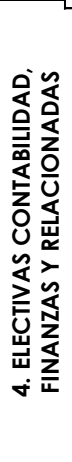 } & 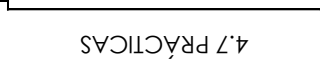 & 0 & ฉ & ○ & 0 & $\stackrel{\sim 0}{-}$ & o & ० & $\wedge$ & $\circ$ & o & 8 & 0 \\
\hline & 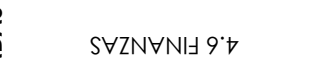 & $\stackrel{4}{\mathrm{~N}}$ & $\infty$ & ৪ & $\overline{0}$ & \pm & $\hat{\jmath}$ & $\circ$ & $\wedge$ & $\circ$ & $\stackrel{t}{0}$ & $\therefore$ & $\stackrel{\circ}{-}$ \\
\hline & $\forall I y O \Perp I \cap \forall s^{\prime} \forall$ & $\stackrel{\sim}{i}$ & $\wedge$ & 20 & fे & $\stackrel{\Xi}{ \pm}$ & io & స్ & $\circ$ & $\stackrel{m}{\dot{m}}$ & $\stackrel{2}{0}$ & $\therefore$ & $\stackrel{\sim}{-}$ \\
\hline & $\mathrm{OHO} \exists \mathrm{d} \exists \triangle \nabla^{\circ} \downarrow$ & $\stackrel{\forall}{\circ}$ & \pm & ৪ & 0 & $\stackrel{n}{2}$ & $\circ$ & $\overline{0}$ & $\circ$ & $\Xi$ & $\circ$ & ㅇ & $\circ$ \\
\hline & 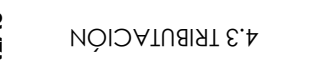 & $\stackrel{\infty}{-}$ & $\infty$ & ㅇ & 0 & $\underline{\sim}$ & $\circ$ & $\overline{0}$ & $\circ$ & \pm & 每 & 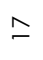 & $\stackrel{\circ}{2}$ \\
\hline & 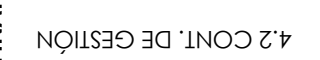 & $\stackrel{\infty}{\circ}$ & $\simeq$ & 아 & 0 & $\stackrel{n}{-}$ & $\circ$ & $\overline{0}$ & $\circ$ & \pm & $\overline{0}$ & $a$ & $\infty$ \\
\hline & 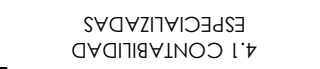 & $\stackrel{m}{+}$ & 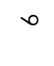 & R & 0 & $\underline{n}$ & $\circ$ & $\stackrel{m}{0}$ & $\circ$ & $\Xi$ & $\stackrel{m}{0}$ & $\stackrel{\infty}{\infty}$ & 으 \\
\hline \multirow{10}{*}{ 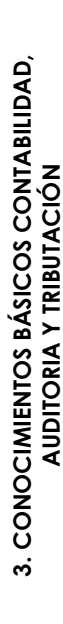 } & $\begin{aligned} \text { OING } \\
7 \exists \triangle N O I\end{aligned}$ & $\stackrel{\circ}{\circ}$ & 0 & R & $\stackrel{a}{-}$ & $\circ$ & 8 & $\overline{0}$ & 0 & \pm & กั & $\stackrel{\circ}{\circ}$ & i \\
\hline & $S \forall Z N \forall N I \exists 6^{\circ} \varepsilon$ & $\stackrel{q}{\dot{\tau}}$ & 0 & 음 & 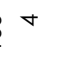 & $\circ$ & 8 & மீ & $\circ$ & 8 & $\underset{i n}{\forall}$ & $\circ$ & 8 \\
\hline & $\forall$ : $\forall I S \forall g \forall \mid \forall O \Perp I O \cap \forall 8^{\cdot} \varepsilon$ & $\stackrel{\sim}{+}$ & 0 & 음 & $\stackrel{m}{m}$ & $\circ$ & 8 & 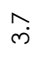 & $\circ$ & ○ & $\stackrel{n}{m}$ & $\circ$ & 8 \\
\hline & ОНОヨУヨ๐ $L^{\circ} \varepsilon$ & $\stackrel{m}{+}$ & $\circ$ & 음 & $\stackrel{m}{+}$ & $\circ$ & $\underline{-}$ & $m$ & $\circ$ & ○ & $\stackrel{m}{+}$ & $\circ$ & 8 \\
\hline & 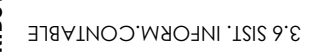 & $\stackrel{\circ}{-}$ & $\circ$ & 8 & - & $\circ$ & $\underline{8}$ & $\cong$ & - & $\infty$ & $\stackrel{\circ}{\longrightarrow}$ & - & $\stackrel{\leftrightarrow}{\alpha}$ \\
\hline & 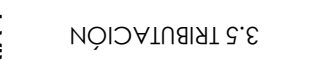 & $\stackrel{\circ}{+}$ & $\circ$ & 음 & $\stackrel{n}{n}$ & $\circ$ & 음 & $\stackrel{\leftrightarrow}{\dot{r}}$ & $\circ$ & ○ & n & $\circ$ & 은 \\
\hline & 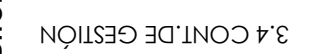 & $\stackrel{\sim}{m}$ & $\circ$ & 8 & $\hat{i}$ & $\circ$ & $\underline{8}$ & $\stackrel{\infty}{\infty}$ & $\circ$ & 8 & $\stackrel{m}{m}$ & 0 & 으 \\
\hline & $\forall O \forall Z$ ZN $\forall \wedge \forall^{\prime} \perp N O \supset \varepsilon^{*} \varepsilon$ & $\hat{\circ}$ & $=$ & $\stackrel{\text { ? }}{q}$ & $\overline{0}$ & 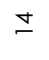 & $\hat{\jmath}$ & $\stackrel{+}{\circ}$ & n & à & $\stackrel{0}{\circ}$ & $a$ & 只 \\
\hline & $\forall y \exists I O N \forall N \mid \exists \cdot I N O \supset$ 乙'E & $\stackrel{+}{+}$ & 0 & 요 & in & $\circ$ & $\underline{8}$ & $\checkmark$ & $\circ$ & ○ & $\stackrel{+}{\stackrel{十}{*}}$ & $\circ$ & 음 \\
\hline & 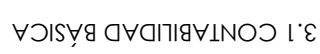 & $\stackrel{m}{\sim}$ & $\circ$ & 요 & - & $\circ$ & 음 & $=$ & - & $\infty$ & $\stackrel{\circ}{-}$ & - & $\stackrel{2}{\alpha}$ \\
\hline $\begin{array}{l}\mathrm{U} \\
\mathbf{N}\end{array}$ & $\mathrm{Z}$ & $\stackrel{\circ}{-}$ & $\circ$ & 8 & $\stackrel{m}{i}$ & - & $m$ & - & $N$ & $\pi$ & & 0 & R \\
\hline \multirow{7}{*}{ 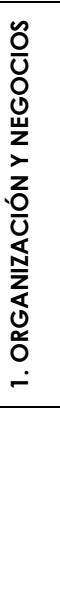 } & STNX $\exists I N I^{\circ}$ & $\stackrel{m}{0}$ & $\Xi$ & ০े & $\overline{0}$ & \pm & $\hat{\circ}$ & $\stackrel{m}{0}$ & $n$ & à & No & $\stackrel{\circ}{\circ}$ & i \\
\hline & $\forall I N O \exists \perp O Q \forall J \forall \exists W G^{*}$ l & $\stackrel{a}{\circ}$ & n & $\stackrel{n}{n}$ & : & $\infty$ & f & $\stackrel{\circ}{\circ}$ & $m$ & in & & $a$ & 织 \\
\hline & 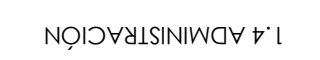 & $\stackrel{n}{n}$ & $m$ & $\stackrel{\sim}{\infty}$ & $\stackrel{n}{\text { in }}$ & - & ळ & $\stackrel{a}{-}$ & $\circ$ & ○ & & 0 & R \\
\hline & $\forall S \exists d d W \exists \forall\urcorner \varepsilon^{\prime} l$ & $\stackrel{\sim}{\infty}$ & $\circ$ & 음 & $\stackrel{\sim}{\sim}$ & $\circ$ & 음 & $\stackrel{\alpha}{\dot{\gamma}}$ & $\circ$ & $\varnothing$ & it & $\circ$ & 으 \\
\hline & 'SヨلdW & $\underset{\sim}{\sim}$ & - & $\stackrel{2}{\alpha}$ & $\stackrel{\sim}{\sim}$ & $\circ$ & 으 & $\stackrel{m}{m}$ & - & $\infty$ & & $m$ & $\stackrel{\sim}{\infty}$ \\
\hline & $\forall !$ WONOOヨ I’l & $\stackrel{a}{i}$ & $\circ$ & 요 & $\stackrel{\infty}{\infty}$ & $\circ$ & $\underline{8}$ & $\hat{i}$ & $\circ$ & 8 & $\hat{i}$ & o & ○ \\
\hline & $\tilde{\underline{m}}$ & 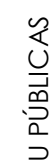 & Z & 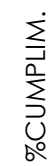 & $\begin{array}{l}\tilde{O} \\
\text { 点 } \\
\underline{\underline{5}}\end{array}$ & z & 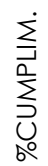 & $\begin{array}{l}+ \\
\stackrel{\alpha}{\alpha} \\
\supset\end{array}$ & Z & 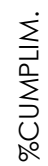 & 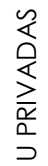 & Z & 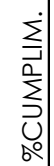 \\
\hline
\end{tabular}

NN = NÚMERO DE IES DE LA MUESTRA QUE NO CUMPLEN CON ASIGNATURAS DEL MÓDELO.

\%CUMPLIM.= PORCENTAJE DE CUMPLIMIENTO DE LAS IES RESPECTO A LAS ASIGNATURAS DEL MODELO.

educade, no 7, 2016, p. 15 


\section{CONSIDERACIONES FINALES}

Ante una propuesta formal, como lo es el modelo de la UNCTAD, las IES mexicanas no han respondido de manera puntual, ya que del análisis del contraste de la muestra de PE contra el modelo, podemos indicar que la tendencia general no es hacia la internacionalización, sino se sigue orientando éstos a panoramas nacionales. Empero los cambios realizados para el último periodo escolar por algunas IES de las más importantes en México, nos hacen pensar en una respuesta que hará que otras IES les secundan.

Encontramos que en los Institutos públicos se da una tendencia a la especialización a la Auditoria o Tributación. En cuanto a conocimientos básicos el resultado muestra que, a pesar de que se cubren plenamente, falta incluir actividades que permitan al alumno integrar los conocimientos adquiridos y que faciliten la comprensión de la función que desempeña el Contador, así como la adquisición de aptitudes tales como la comunicación verbal y escrita y a comprender y resolver problemas reales y concretos.

Una gran excepción en cuanto a cumplimiento con el modelo es el caso de las asignaturas electivas, donde encontramos que cumplen realmente muy pocas IES, entendiéndose el caso en las IES pequeñas, dado el costo que supondría ofertar este tipo de electivas, sin embargo en las Universidades públicas ha faltado una estrategia que permita cumplir con la propuesta.

De las limitaciones en este trabajo podemos señalar, primero, que sólo contrasta los temas acerca de la preparación técnica no así las habilidades y los valores. Así mismo, al no publicar suficiente información las IES en internet, nos vimos obligados a realizar el contraste con el número de asignaturas dedicadas a cada módulo y no con el número de créditos, que nos daría un número más puntual de horas/clase dedicadas a cada tema. Otra limitación que encontramos, que afecta un poco el resultado es la denominación de las asignaturas, es que encontramos alguna dificultad al clasificarlas con seguridad, al no publicar las IES el contenido de la asignatura en línea.

\section{BIBLIOGRAFÍA}

Arquero, J.L., Donoso-Anes, J.A., Jiménz-Cardoso, S.M., González, J.M., (2009). Análisis exploratorio del perfil demandado para Administración y Dirección de Empresas: implicaciones para el área contable. Revista de Contabilidad. España. Vol. 12 No. 2. 192.

Boritz, J.E. (1999). The Accounting Curriculum and IT. Scholl of Accountacy, Universidad de Waterloo. Canadá. 4.

Brambila, L. y Vázquez, S. (2006). Las competencias profesionales del Contador Público, base para una inserción laboral exitosa. Contaduría Pública. México. Sep. 3637.

Gómez, B., Puig, L., Quirós, A. y Viaño, J.M. (s.f.) La Convergencia Europea en Educación y las Nuevas Leyes Educativas Españolas LOU y LOCE. Recuperado el 27 de junio de 2011, de http://www.uv.es/gomezb/37Laconvergenciaeuropea.pdf 
Gracia Patricia Michel, Carmen Fernández-Polvillo

El modelo de plan de estudios de la UNCTAD propuesto para el profesional en la Contaduría versus la estructura curricular de las Universidades mexicanas: un estudio comparativo

Guía metodológica para la evaluación de los currícula de licenciatura (1992). Comités Interinstitucionales para la Evaluación de la Educación Superior. México. Recuperado el 12 de noviembre de 2011 de http://www.ciees.edu.mx/ciees/publicaciones.

International Federation of Accountants (2010). "Competent and Versatile: Q\&A for Smaller Organizations and the Accountants That Serve Them" (Elaborado por la Professional Accountants in Business Committee). Recuperado el 15 de noviembre de 2011 de http://web.ifac.org/download/Competent_Versatile_QAs_SMEs.pdf

International Federation of Accountants (2010). Competent and Versatile: How Professional Accountants in Business Drive Sustainable Organizational Success (Elaborado por la Professional Accountants in Business Committee). Recuperado el 15 de noviembre de 2011 de http://www.ifac.org/Guidance/EXD-Details.php?EDID=0142

International Federation of Accountants (2010). Handbook of International Education Pronouncements (Elaborado por la International Accounting Education Standards Board). Recuperado el 15 de noviembre de 2011 de http://web.ifac.org/publications/international-accounting-educationstandards-board/handbook

Ley General de Educación (1993). Última reforma DOF 9-04-2012. México. Recuperado el 18 de abril de 2012 de http://www.diputados.gob.mx/LeyesBiblio/pdf/137.pdf

Meljem, S. (2006). Entrevista con el C.P. Víctor Everardo Beltrán Corona, Director General de Profesiones de la SEP. Contaduría Pública. México. Sep. 6-9.

Oliveira, A., Veneroso, J., Carvalho de Souza, M.J. (2009) Análise comparativa entre os currículos dos cursos de Ciencias Contábeis das Universidades do Estado de Santa Catarina listadas pelo MEC e o currículo mundial proposto pela ONU/UNCTAD/ISAR. Gestao \& Regionalidade. Brasil. Vol. 25 No. 75. 22-30.

Organización para la Cooperación y el Desarrollo Económico (2009). 21 st Century Skills and Competences for New Millennium Learners in OECD Countries. (EDU Working paper no. 41OECD Publishing.doi: 10.1787/218525261154). P. 6.

Ott, E., Barbosa, Ch. (2010) Estrutura curricular do curso de Ciéncias Contábeis no Brasil versus estruturas curriculares propostas por organismos internacionais: uma análise comparativa. Universo Contábil. Brasil. Vol. 6 No.1. 28-45.

Romero, R. (2006). ¿Será cierto que la carrera de Contaduría Pública presenta "rendimientos" marginales decrecientes tales que está en riesgo su futuro?. Contaduría Pública. México. Sep. 10-11.

United Nations Conference on Trade and Development/UNCTAD. 1999. Directiva para la Elaboración de un Programa Mundial de Estudios de Contabilidad y otras Normas y Requisitos de Cualificación. TD/B/COM.2/ISAR/5. Recuperado el 15 de noviembre de 2011 de http://unctad.org/es/docs/c2isard5.sp.pdf

United Nations Conference on Trade and Development/UNCTAD. 2003. Informe del Grupo de Trabajo Intergubernamental de Expertos en Normas Internacionales de Contabilidad y Presentación de Informes sobre su Vigésimo Periodo de Sesiones. TD/B/COM.2/58 TD/B/COM.2/ISAR/22. Recuperado el 15 de noviembre de 2011 de 
El modelo de plan de estudios de la UNCTAD propuesto para el profesional en la Contaduría versus la estructura curricular de las Universidades mexicanas: un estudio comparativo

http://archive.unctad.org/Templates/Meeting.asp? intltemID=1942\&lang=1\&m $=5992 \&$ year $=2003 \&$ month $=1$

United Nations Conference on Trade and Development/UNCTAD. 2003. Guideline on national requeriments for the qualification of the professional accountants. Recuperado el 15 de noviembre de 2011 de http://archive.unctad.org/Templates/Meeting.asp? intltemID=1942\&/ang $=1 \& \mathrm{~m}$ $=5992 \&$ year $=2003 \&$ month $=1$ 